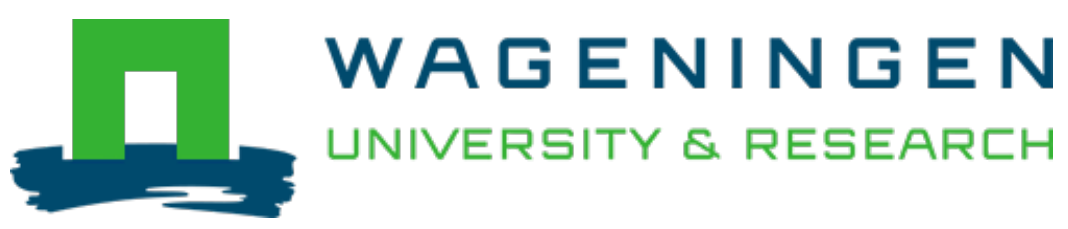

\title{
SNP genotyping in tetraploid cut roses
}

\author{
Acta Horticulturae \\ Koning-Boucoiran, C.F.S.; Smulders, M.J.M.; Krens, F.A.; Esselink, G.D.; Maliepaard, C.A. \\ https://doi.org/10.17660/ActaHortic.2012.953.49
}

This article is made publicly available in the institutional repository of Wageningen University and Research, under the terms of article $25 \mathrm{fa}$ of the Dutch Copyright Act, also known as the Amendment Taverne. This has been done with explicit consent by the author.

Article 25 fa states that the author of a short scientific work funded either wholly or partially by Dutch public funds is entitled to make that work publicly available for no consideration following a reasonable period of time after the work was first published, provided that clear reference is made to the source of the first publication of the work.

This publication is distributed under The Association of Universities in the Netherlands (VSNU) 'Article $25 \mathrm{fa}$ implementation' project. In this project research outputs of researchers employed by Dutch Universities that comply with the legal requirements of Article $25 \mathrm{fa}$ of the Dutch Copyright Act are distributed online and free of cost or other barriers in institutional repositories. Research outputs are distributed six months after their first online publication in the original published version and with proper attribution to the source of the original publication.

You are permitted to download and use the publication for personal purposes. All rights remain with the author(s) and / or copyright owner(s) of this work. Any use of the publication or parts of it other than authorised under article $25 \mathrm{fa}$ of the Dutch Copyright act is prohibited. Wageningen University \& Research and the author(s) of this publication shall not be held responsible or liable for any damages resulting from your (re)use of this publication.

For questions regarding the public availability of this article please contact openscience.library@,wur.nl 


\title{
SNP Genotyping in Tetraploid Cut Roses
}

\author{
C.F.S. Koning-Boucoiran ${ }^{\mathrm{a}}$, M.J.M. Smulders, F.A. Krens, \\ G.D. Esselink and C. Maliepaard \\ WUR Plant Breeding \\ PO Box 16, 6700 AA Wageningen \\ The Netherlands
}

Keywords: Rosa hybrida, cut roses, tetraploid inheritance, powdery mildew resistance, QTL, SNP marker, marker assisted breeding

\begin{abstract}
Genetic analysis in tetraploid rose is complex: inheritance of genes could be disomic, keeping the two original diploid genomes separate but it could also be tetrasomic, permitting the four homologous chromosomes to pair and recombine during meiosis, and including the possibility of double reduction. Marker-assisted breeding (MAB) is particularly suited for traits such as disease resistance since disease resistance assays are laborious, time and money consuming, but we need to extend the number of markers for better coverage of the genome before being able to find associations and apply MAB. Our goal was to produce hundreds of single nucleotide polymorphism (SNP) markers to provide the coverage needed. Disease resistance, more specifically powdery mildew resistance is our primary target because chemical pest control will be increasingly limited. Molecular markers could provide a good tool to facilitate selection in an earlier stage of a breeding program. We investigated the inheritance of powdery mildew resistance in an $F_{1}$ population originating from a cross between two tetraploid genotypes, P540 and P867, differing in powdery mildew resistance. SNP marker development started with Next Generation Sequencing (Illumina GAII) of cDNA obtained from RNA isolated from flowers at three developmental stages for each parent. We identified a large number of putative single nucleotide polymorphisms (SNPs) between and within both parents. SNPs that were expected to segregate as simplex $\times$ nulliplex and duplex $\times$ nulliplex markers were selected for genotyping the entire $F_{1}$ population using the KASP platform (KBioscience). For a pilot study on a limited number of SNPs we defined specific selection criteria resulting in a set of 20 SNPs for testing on the population. It was found that selecting the SNPs according to the ratio of the number of reads from the Illumina sequencing data did not guarantee the appropriate segregation ratios in the progeny. Continued research will focus on identifying haplotypes and on QTL mapping of powdery mildew resistance. We will present results on the criteria used to select SNP markers, success rate, the realised segregation ratios in the offspring, the reconstruction of the homologous chromosomes (haplotypes) in the cross, and QTL mapping of powdery mildew resistance.
\end{abstract}

\section{INTRODUCTION}

Tetraploid Hybrid Tea roses (Rosa hybrida) were obtained through interspecific hybridizations between about 10 different diploid and tetraploid rose species. Nowadays, they represent most of the commercial cut roses and form the basis of breeding programmes. Newly obtained cultivars still hardly combine the desired aesthetic traits with economically important traits such as powdery mildew resistance. Powdery mildew, caused by the pathogenic fungus Podosphaera pannosa, results in major yield losses during the production of cut roses. This obligate parasite reduces the quality of the flowers which make them unsuitable to sell, leading to gain losses. Therefore nearly $40 \%$ of the fungicides sprayed on roses is to control powdery mildew (Linde and Shishkoff,

a carole.boucoiran@wur.nl 
2003). Moreover, a high diversity in virulence among powdery mildew isolates, and differential host responses have been reported (Linde and Debener, 2003; Leus, 2005; Yan et al., 2006). Linde and Debener (2003) showed that powdery mildew resistance was controlled by a single dominant resistance gene called Rppl in diploid roses. Hosseini Moghaddam et al. (2012) detected 9 QTL loci on four different linkage groups in their diploid population. Later, Yan (2005) and Koning-Boucoiran et al. (2012) reported the polygenic nature of powdery mildew resistance in tetraploid roses.

Due to the tetraploid nature of cut roses and to the high level of heterozygosity, it is difficult to control and predict the genetic inheritance to combine a number of desired traits. Transmission of genes can follow a disomic inheritance keeping the two original diploid genomes separate, but it can also follow a tetrasomic inheritance permitting the four homologous chromosomes to pair and recombine during meiosis, including the possibility of double reduction. Therefore, tetraploidy may lead to a complex inheritance pattern for which marker assisted breeding (MAB) would help to direct selection. MAB is particularly suited for traits such as disease resistance since disease resistance assays are laborious, time and money consuming, but to find such markers we first need to extend the number of markers for better coverage of the genome. The available genetic linkage maps of tetraploid cut roses (Koning-Boucoiran et al., 2012) has a coverage of one marker every six $\mathrm{cM}$ which is still too low to find strong association with interesting traits. Production of hundreds of single nucleotide polymorphism (SNP) markers could provide the coverage needed for the identification of allele-specific markers linked to disease resistance allowing precision breeding.

Here, we will present results on the criteria used to select SNP markers, success rate, the realised segregation ratios in the offspring, the reconstruction of the homologous chromosomes (haplotypes) in the cross, and QTL mapping of powdery mildew resistance.

\section{MATERIALS AND METHODS}

The tetraploid rose population K5 that Yan (2005) investigated in this study consisted of the offspring of a cross between two tetraploid genotypes P540 (mother) and P867 (father) from a cut rose hybrid tea breeding program. P540 is a commercial cultivar developed at Terra Nigra B.V. (The Netherlands) with dark red flowers (Fig. 1A). P867 displays pale salmon coloured flowers (Fig. 1B) and is more resistant to powdery mildew. The segregating progeny consisting of 184 genotypes, was planted in a heated sun-lit greenhouse at $20 / 17^{\circ} \mathrm{C}$ (day/night), a day length of $18 \mathrm{~h}$, and a relative humidity of ca. $80 \%$. The experiment had a randomized complete block design with two replicates, each replicate consisting of two plants per genotype. Powdery mildew resistance data were obtained from Yan (2005) and Yan et al. (2006) who tested the K5 population for resistance against two monospore isolates (2 and F1) of Podosphaera pannosa (Wallr.:Fr.) de Bary (syn. Sphaerotheca pannosa).

Petals of flowers at three different stages (S1, S2, S3) were harvested for both parents (Fig. 2). The purification of total RNA was performed using the RNeasy Plant Mini Kit (QIAGEN, Westburg, The Netherlands) according to the manufacturer's instructions. Total RNAs of all three flowers stages were pooled, and sent to be converted into cDNA and subsequently sequenced using the Illumina platform (GAII, ServiceXS, Leiden, The Netherlands). A de novo assembly of each parent was performed using Trinity (Grabherr, 2011) and the resulting transcripts were assembled to a consensus transcriptome using CAP3 (Huang, 1999). Subsequently, the reads of each parent were mapped against the consensus transcriptome using Bowtie2 (Langmead and Salzberg, 2012). QualitySNP (Tang et al., 2006) was used to identify reliable single nucleotide polymorphisms (SNPs). The transcripts were also blasted against the Genome Database for Rosaceae for annotation. A set of 20 SNPs was selected for testing with a competitive allele specific assay (KASP, KBioscience) on the whole population in a pilot study. The following criteria were used to select the SNPs: number of reads higher or equal to 25 , only two nucleotides involved in the SNP, ratio between the major and the minor allele should be $3: 1$ or $1: 1$, minor allele present in only one parent, and flanking regions of 
50 nucleotides free from SNPs. We also selected SNPs in contigs which were annotated.

The results of the KASP genotyping were visualized with the program SNPviewer (v. 1.99.1, KBioscience).

\section{RESULTS AND DISCUSSION}

Out of the 20 SNPs genotyped on 175 individuals of the K5 population, one SNP marker did not show any product amplification and two appeared to be monomorphic for both parents and did not show any segregation. For two SNPs, the segregation could not be explained because it was too far from any expected segregation, which could be due to the presence of null alleles. For one SNP, the progeny showed a 3:1 segregation $(p=0.34$, $d f=1)$ which would be expected for a simplex $\times$ simplex $(\mathrm{S} \times \mathrm{S})$ marker if scored as dominant, so without distinguishing single or double dosage of the allele. However, it was not possible to distinguish the different phenotypic classes according to dosage. Twelve SNPs were not significantly different from a 1:1 simplex $\times$ nulliplex $(S \times N)$ segregation (Table 1). For those SNPs, the progeny was clustered in two distinct groups displaying respectively an homozygous genotype and an heterozygous genotype. For two SNPs, the progeny showed a segregation in agreement with $35: 1(\mathrm{p}>0.70, d f=1)$ which would be typical for a duplex $\times$ duplex marker, if scored as dominant, without distinguishing different dosages of the alleles. For one of them, it was not possible to distinguish the underlying different dosage classes. For the other one, it was possible to distinguish the five dosage classes which are typical for a 35:1 segregation. Those classes were not significantly different from a 1:8:18:8:1 segregation expected for nulliplex, simplex, duplex, triplex, quadruplex presence of an allele $(\mathrm{p}=0.99, d f=4)$. These five classes could not be separated using the SNPViewer software since the clustering within this programme is for diploid species. However, the classes could be manually inferred (Fig. 3). Both parents would belong to the same duplex dosage class GGAA which would fit to a duplex $\times$ duplex segregation. But, they are not grouped in the correct dosage class because their signal intensity might be too low for analysis or they were not properly genotyped. Since the automatic assignation of genotypes from SNPViewer cannot be used to assign dosage of the alleles, the data of the complete SNP set will be analysed with the programme fitTetra (Voorrips et al., 2011). This will help the genetic analysis and the interpretation in terms of disomic, tetrasomic or intermediate inheritance. Our tetraploid population showed some evidence for an intermediate inheritance mode between disomic and tetrasomic for markers (SSRs) that were previously tested (Koning-Boucoiran et al., 2012) but with the SNPs it should be possible to shed more light on the segregational pattern.

The results from the preliminary selection of 20 SNPs showed that selecting the SNPs according to the ratio of the number of reads between the major and the minor allele (e.g., 1:1 in case of $S \times N$, or 3:1 in case of $S \times S$ ) from the Illumina sequencing data did not guarantee a 1:1 or a 3:1 segregation respectively in the progeny. Therefore, the complete set of SNPs was subsequently subjected to a screening according to the criteria mentioned in Materials and Methods. Moreover, SNPs were selected in order to enable identification of haplotypes. For this, SNPs characterizing the different haplotypes (preferentially four) within a contig were chosen. The results of genotyping the population with the total set of SNPs will be presented at the symposium as well as the identification of the different haplotypes when possible. A QTL analysis including the new SNP markers will be performed in order to map QTLs for powdery mildew resistance which can be used for MAB.

\section{ACKNOWLEDGEMENTS}

We thank Terra Nigra B.V. (The Netherlands) for generating the K5 population and making it available. This research was funded in part by TTI Green Genetics.

Literature Cited

Grabherr, M.G., Haas, B.J., Yassour, M., Levin, J.Z., Thompson, D.A., Amit, I., 
Adiconis, X., Fan, L., Raychowdhury, R., Zeng, Q., Chen, Z., Mauceli, E., Hacohen, N., Gnirke, A., Rhind, N., di Palma, F., Birren, B.W., Nusbaum, C., Lindblad-Toh, K., Friedman, N. and Regev, A. 2011. Full-length transcriptome assembly from RNA-Seq data without a reference genome. Nature Biotech. 29:644-652.

Hosseini Moghaddam, H., Leus, L., De Riek, J., Van Huylenbroeck, J. and Van Bockstaele, E. 2012. Construction of a genetic linkage map with SSR, AFLP and morphological markers to locate QTLs controlling pathotype-specific powdery mildew resistance in diploid roses. Euphytica 184:413-427.

Huang, X. and Madan, A. 1999. CAP3: A DNA Sequence Assembly Program. Genome Res. 9:868-877.

Koning-Boucoiran, C.F.S., Gitonga, V., Yan, Z., Dolstra, O., van der Linden, C.G., van der Schoot, J., Uenk, G., Verlinden, K., Smulders, M.J.M., Krens, F.A. and Maliepaard, C. 2012. The mode of inheritance in tetraploid cut roses. TAG. doi.10.1007/s00122-012-1855-1.

Langmead, B. and Salzberg, S. 2012. Fast gapped-read alignment with Bowtie 2. Nature Methods 9:357-359.

Leus, L. 2005. Resistance breeding for powdery mildew (Podosphaera pannosa) and black spot (Diplocarpon rosae) in roses. Ph.D. Thesis, Faculty of Bioscience Engineering, Ghent University, Belgium. 149p.

Linde, M. and Debener, T. 2003. Isolation and identification of eight races of powdery mildew of roses (Podosphaera pannosa) (Wallr.: Fr.) de Bary and the genetic analysis of the resistance gene Rpp1. TAG 107:256-262.

Linde, M. and Shishkoff, N. 2003. Powdery mildew. p.158-165. In: A.V. Roberts, T. Debener and S. Gudin (eds.), Encyclopedia of rose science. Elsevier, Oxford.

Tang, J., Vosman, B., Voorrips, R.E., van der Linden, C.G. and Leunissen, J.A. 2006. QualitySNP: a pipeline for detecting single nucleotide polymorphisms and insertions/deletions in EST data from diploid and polyploid species. BMC Bioinformatics 7:438.

Voorrips, R.E., Gort, G. and Vosman, B. 2011. Genotype calling in tetraploid species from bi-allelic marker data using mixture models. BMC Bioinformatics 12:172.

Yan, Z. 2005. Towards efficient improvement of greenhouse grown roses: genetic analysis of vigour and powdery mildew resistance. PhD. Thesis. WUR, The Netherlands.

Yan, Z., Dolstra, O., Prins, T., Stam, P. and Visser, P. 2006. Assessment of partial resistance to powdery mildew (Podosphaera pannosa) in a tetraploid rose population using a spore-suspension inoculation method. European Journal of Plant Pathology 114:301-308. 


\section{Tables}

Table 1. List of the tested SNPs which are not significantly different from a 1:1 segregation $(\mathrm{SxN})$ according to a Chi-square goodness-of-fit test at $d f=1$.

\begin{tabular}{lcccccc}
\hline SNP name & $\begin{array}{c}\text { SNP } \\
\text { polymorphism }\end{array}$ & Heterozygous & Homozygous & $\begin{array}{c}\text { Not } \\
\text { classified }\end{array}$ & Ratio & P $d f=1$ \\
\hline P540_S002 & AG/GG & 94 & 78 & 1 & 0.8 & 0.64 \\
P540_S004 & CT/TT & 67 & 89 & 17 & 0.8 & 0.78 \\
P540_S008 & TG/GG & 66 & 91 & 16 & 0.7 & 0.83 \\
P540_D001 & AG/GG & 80 & 92 & 1 & 0.9 & 0.55 \\
P540_D002 & CT/TT & 80 & 92 & 1 & 0.9 & 0.55 \\
P867_S009 & GA/AA & 79 & 55 & 38 & 0.7 & 0.85 \\
P867_S010 & TC/CC & 93 & 76 & 4 & 0.8 & 0.66 \\
P867_S011 & TA/AA & 91 & 77 & 5 & 0.8 & 0.60 \\
P867_S012 & CG/GG & 91 & 72 & 10 & 0.8 & 0.71 \\
P867_S014 & GC/CC & 78 & 91 & 4 & 0.9 & 0.57 \\
P867_S015 & GC/CC & 73 & 97 & 3 & 0.8 & 0.80 \\
P867_SS002 & CT/TT & 77 & 80 & 16 & 1.0 & 0.37 \\
\hline
\end{tabular}

$\underline{\text { Figures }}$

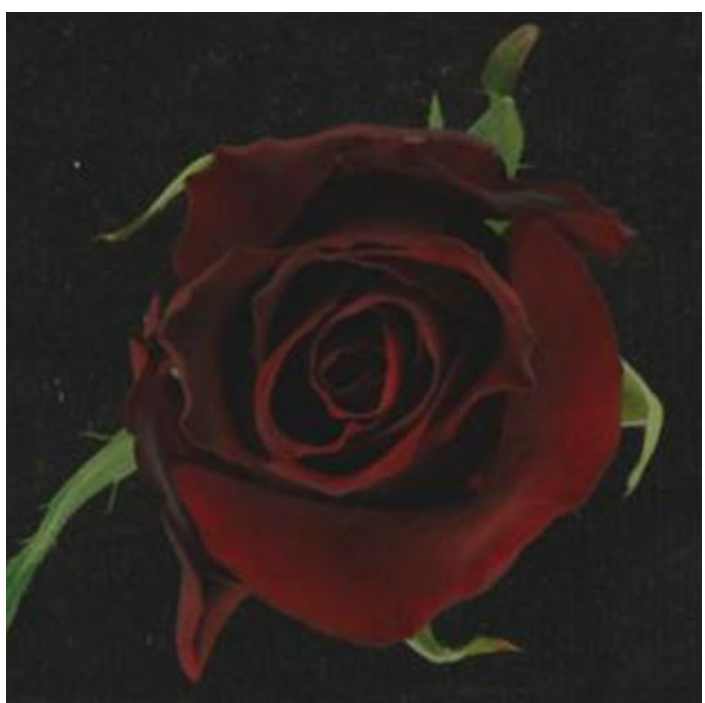

A

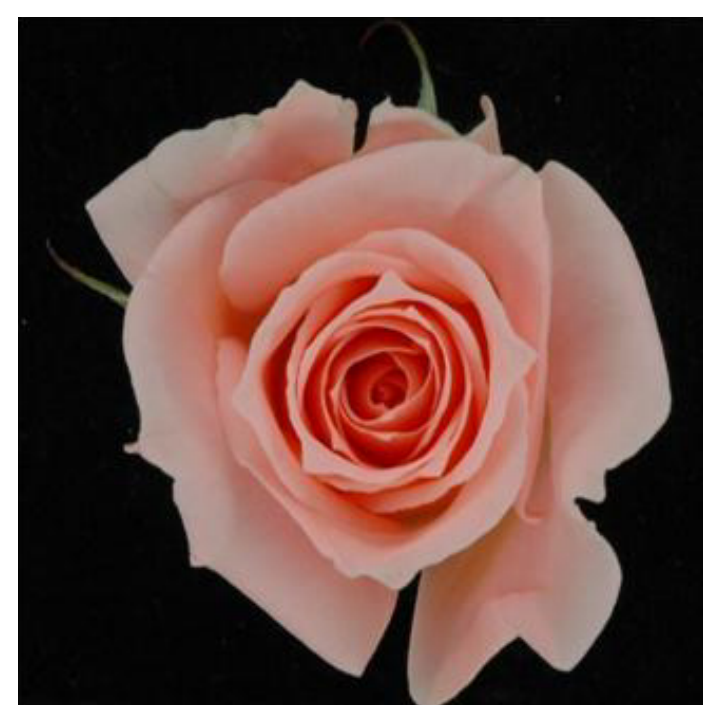

B

Fig. 1. Picture of the flowers of the parents of the K5 population. A: P540 (mother). B: P867 (father). 


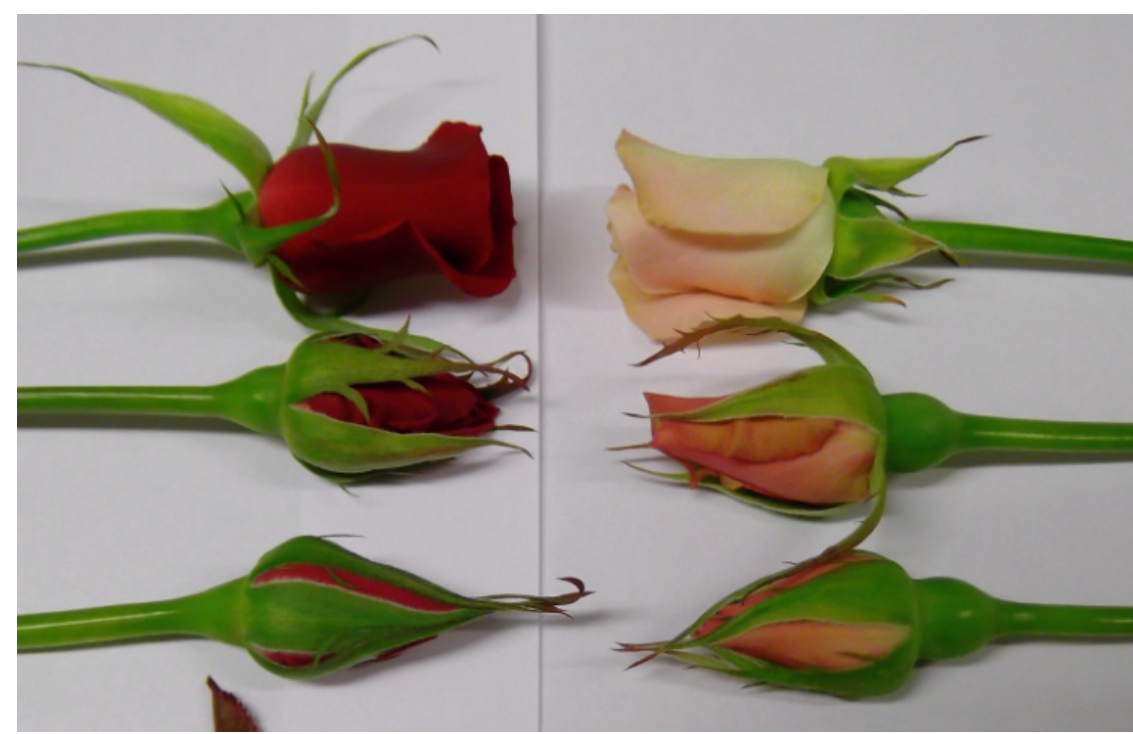

S3

S2

A

B

Fig. 2. Different developmental stages (S1, S2, S3) of the harvested flowers for both parents P540 (A) and P867 (B).

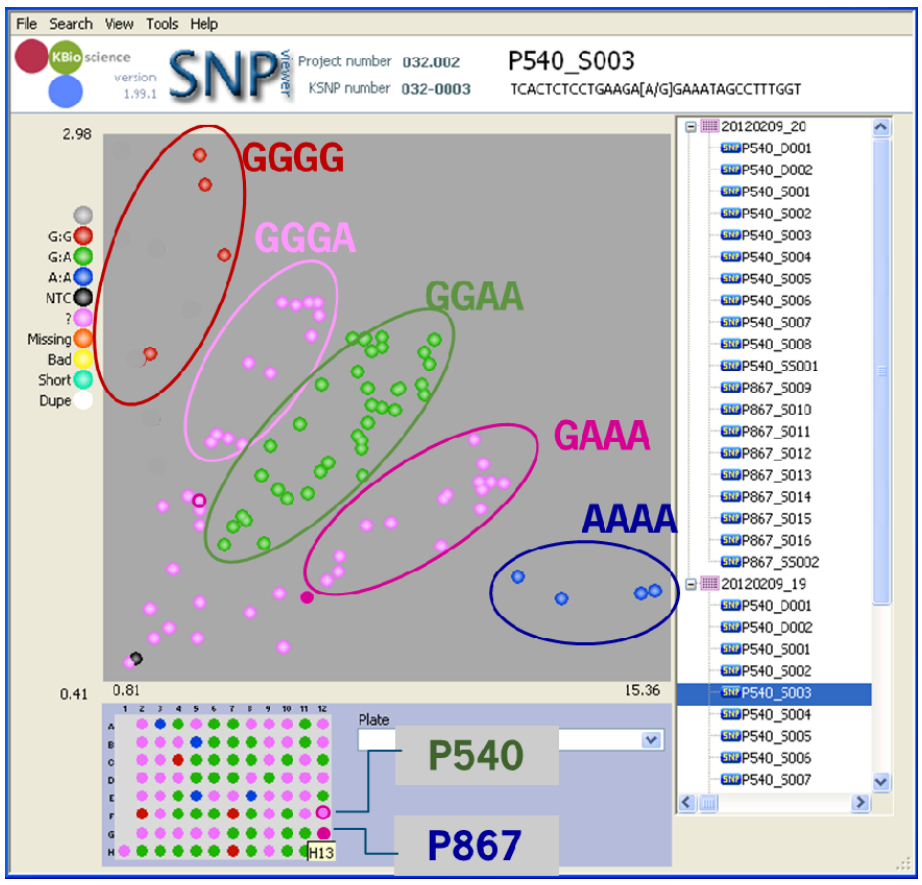

Fig. 3. Graphical representation with SNPviewer and the interpretation in five dosage classes in case of a duplex $\times$ duplex SNP marker showing a 1:8:18:8:1 segregation. The five classes were manually inferred according to the expected segregation. P540: pink open circle. P867: pink plain circle. 